\title{
PROOF VALIDATION AND MODIFICATION BY EXAMPLE GENERATION: A CLASSROOM-BASED INTERVENTION IN SECONDARY SCHOOL GEOMETRY
}

\author{
Kotaro Komatsu $^{1}$, Tomoyuki Ishikawa ${ }^{2}$, and Akito Narazaki ${ }^{3}$ \\ ${ }^{1}$ Shinshu University, Japan; ${ }^{2}$ Nagano Municipal High School, Japan; ${ }^{3}$ Tojaku High School, Japan
}

This study addresses proof validation and modification in geometry wherein students find cases invalidating their constructed proofs and revise their proofs in response to this invalidation. We implemented a classroom-based intervention in grade 9, in which the students worked on assessment tasks designed to assess their performance in proof validation and modification at the end of the intervention. After reporting the intervention in brief, this study analyses the results of the tasks to examine the number of students who succeeded in proof validation and modification, the types of diagrams drawn by the students for invalidation of their proof, and their responses to this invalidation.

\section{INTRODUCTION}

The study reported in this paper addresses proof validation (Selden \& Selden, 1995, 2003), which is defined as reading and checking an argument constructed as a proof, to determine whether the argument really constitutes a legitimate proof, that is, whether the argument can establish the truth of a statement. Proof validation is a crucial activity, at not only the undergraduate level, but also primary and secondary school levels, since the Common Core State Standards Initiative (2010) emphasises the ability to critique the reasoning of others as one of the standards for mathematical practice. It can also cultivate students' critical thinking and attitude that are essential attributes of literate citizens. Moreover, explicit instruction in proof validation could enhance students' ability to construct valid proofs (Powers, Craviotto, \& Grassl, 2010).

Existing mathematics education research has investigated actual behaviour regarding proof validation by undergraduate students, teachers, and professional mathematicians (Alcock \& Weber, 2005; Inglis \& Alcock, 2012; Knuth, 2002; Ko \& Knuth, 2013; Segal, 1999; Selden \& Selden, 2003; Weber, 2008, 2010), demonstrating that proof validation is difficult for undergraduate students and teachers. It is therefore easily conjectured that this difficulty would be clearly shown through the work of secondary school students; however, there is little research that explores how these students really engage in proof validation.

In what follows, we illustrate a specific task to introduce proof validation into secondary school geometry and construct a framework for classifying levels of proof validation and modification. After specifying the research questions, this paper reports on a classroom-based intervention implemented to explore how secondary school students work on proof validation and modification.

\section{PROOF VALIDATION AND MODIFICATION IN SECONDARY SCHOOL GEOMETRY}

This study uses proof problems with diagrams (Komatsu, Tsujiyama, Sakamaki, \& Koike, 2014) as tasks to introduce proof validation into secondary school geometry. A proof problem with a 
diagram is a problem where a statement is described with reference to a particular diagram with symbols. An example of the tasks is shown in Figure 1, where the statement can be proved by showing $\angle \mathrm{QAC}=\angle \mathrm{PBD}$ and $\angle \mathrm{QCA}=\angle \mathrm{PDB}$ from the inscribed angle theorem.

As shown in the diagram to the right, two circles $\mathrm{O}$ and $\mathrm{O}$ ' intersect at points $\mathrm{P}$ and $\mathrm{Q}$, and two points $\mathrm{A}$ and $\mathrm{B}$ are located on circle $\mathrm{O}$. Point $\mathrm{C}$ is the intersection point of line AP and circle $\mathrm{O}^{\prime}$, and point $\mathrm{D}$ is the intersection point of line $B Q$ and circle $\mathrm{O}^{\prime}$. Prove that $\triangle \mathrm{AQC}$ is similar to $\triangle \mathrm{BPD}$.

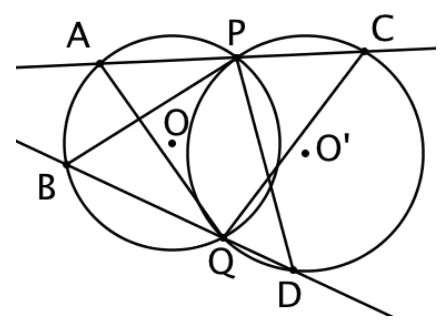

Figure 1: An example of proof problem with diagram

We adopt the specific interpretation of this task where the relevant statement is considered under a certain general class to which the attached diagram belongs, rather than only for the diagram given. For instance, we regard that the statement in Figure 1 argues that $\triangle \mathrm{AQC}$ is always similar to $\triangle \mathrm{BPD}$ if point $\mathrm{A}$ is located on circle $\mathrm{O}$ as shown in Figure 2. This interpretation often enables students to use examples for proof validation, where they draw diagrams satisfying the assumption of the statement and inspect whether a constructed proof is applicable to these diagrams. In the above example, the proof for Figure 1 is invalidated by Figure $2 b$, which rejects the inscribed angle theorem used to deduce $\angle \mathrm{QCA}=\angle \mathrm{PDB}$, and the proof needs to be modified by changing the reason to the inscribed quadrilateral theorem. Following Lakatos (1976), we use the term 'local counterexamples' to refer to examples that reject a constructed proof but not a statement itself.

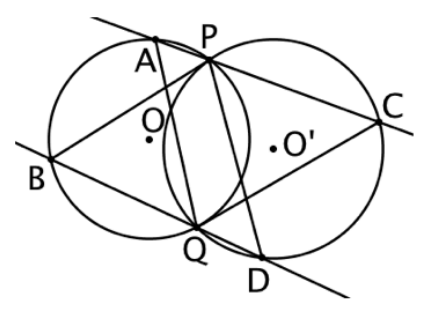

a

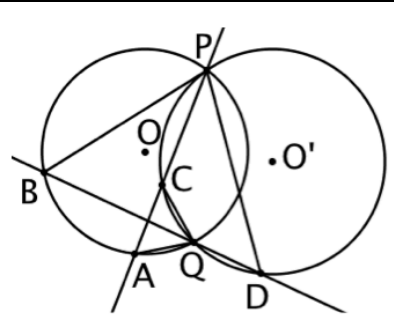

$\mathrm{b}$

Figure 2: Diagrams that satisfy the assumption of the statement

Based on the above illustration, this study constructs the framework shown in Table 1 to classify levels of proof validation and modification. Level 0 refers to failure in discovering a local counterexample, whereas level 1 refers to success in the discovery but failure in modifying a proof to cope with the local counterexample. Level 2 refers to success in the proof modification, and level 3 refers to reaching level 2 and further finding other kinds of local counterexamples.

\begin{tabular}{ccccc}
\hline & Level 0 & Level 1 & Level 2 & Level 3 \\
\hline Proof validation & X & $\checkmark$ & $\checkmark$ & $\checkmark$ (multiple) \\
Proof modification & X & X & $\checkmark$ & $\checkmark$ \\
\hline
\end{tabular}

Table 1: A framework to classify levels of proof validation and modification 
Note that the meaning of proof validation in this study is peculiar in contrast with existing research. The previous studies focused on identifying faults in invalid proofs, such as the falsehood of the reason used to deduce a specific claim, the mismatch of an argument where the converse of a target proposition is proved, and the inappropriateness of the counterexample used to show the falsehood of a proposition (e.g., Alcock \& Weber, 2005; Ko \& Knuth, 2013; Selden \& Selden, 2003). In contrast, this study does not deal with completely invalid proofs, but rather consider proofs that are valid only for certain classes, investigating whether students can discover the cases for which the proofs are not applicable.

Based on the above discussion, this study addresses the following research questions:

- To what extent can secondary school students discover a local counterexample that invalidates their proof?

- To what extent can secondary school students modify their proof so that the modified proof can be valid for the local counterexample that they have discovered?

\section{METHODS}

We decided to investigate student behaviour after classroom-based intervention (Stylianides \& Stylianides, 2013) regarding proof validation and modification. Although students may regularly, but implicitly, scrutinise the validity of their own as well as others' proofs, instruction focusing on proof validation would be uncommon. It implies that students' ability to validate and modify a proof would be limited in ordinary environments. However, some studies suggest that student comprehension and validation of proofs can be improved by relevant instruction (Alcock \& Weber, 2005; Hodds, Alcock, \& Inglis, 2014; Powers et al., 2010; Selden \& Selden, 2003). Hence, more students would succeed in proof validation and modification with explicit instruction, which supports the introduction of this activity into ordinary classrooms.

The classroom-based intervention examined in this paper was implemented under a larger study on curriculum development for explorative proving (Miyazaki \& Fujita, 2015). This intervention aimed at fostering proof validation and modification in secondary school geometry with the theory of hypothetical leaning trajectory (Simon, 1995; Simon \& Tzur, 2004) to develop a set of tasks and relevant instructional actions with close collaboration between researchers and teachers. The second author of this paper implemented a three-hour teaching experiment in his classroom at a lower secondary public school, with 29 Japanese ninth graders (14-15 years old). In the latter half of the third lesson, the students undertook assessment tests designed specifically to address the above research questions. The used tests, which related to Figure 1, will be described later in detail. Prior to the intervention, the participating students had learnt proofs using the conditions for similar triangles and the inscribed angle theorem. Although they had learnt the inscribed quadrilateral theorem, they had not worked on a proof problem that required this theorem. We expected the first lesson to be an effective setting for the students to experience the problem for the first time.

Due to the page limitation, this paper focuses on the analysis of the students' responses to the assessment tasks, and omits the description of the hypothetical learning trajectory and detailed reports of the implemented lessons. These omissions are to lend focus to the purpose of this paper which is not to evaluate the effectiveness of the teaching experiments for the facilitation of proof validation and modification; such evaluation needs a deep analysis into the relationship between the 
teaching experiments and the students' performance on the assessment tasks. Instead, this paper aims to describe to what extent the students can perform proof validation and modification.

All the teaching experiments were videotaped and transcribed, and the students' worksheets were collected. The first author observed all the lessons and took field notes. To analyse the students' answers to the assessment tests, the first author initially created the category shown in Table 1 for classifying these answers. Subsequently, the first author and third author independently classified each student's answers according to this category. We synthesised our classifications and discussed the discrepancies until we reached a consensus.

\section{RESULTS}

\section{The first and second lessons}

The first lesson involved the problem shown in Figure 3, in which the students constructed a proof by showing $\angle \mathrm{BPA}=\angle \mathrm{CPD}$ and $\angle \mathrm{BAP}=\angle \mathrm{CDP}$ from the equality of the vertical angles and the inscribed angle theorem, respectively. The teacher then used GeoGebra to move point $\mathrm{A}$ and present the diagram shown in Figure $3 \mathrm{~b}$, which the students recognised as a local counterexample of their proof because both of the reasons for $\angle \mathrm{BPA}=\angle \mathrm{CPD}$ and $\angle \mathrm{BAP}=\angle \mathrm{CDP}$ were invalidated. Then, the teacher prompted the students to modify their proof, and the students responded by changing the reasons, such as from the equality of the vertical angles to the identity of the angles.

As shown in the right diagram, there are four points $\mathrm{A}, \mathrm{B}, \mathrm{C}$, and $\mathrm{D}$ on circle $\mathrm{O}$. Point $\mathrm{P}$ is the intersection point of lines $\mathrm{AC}$ and $\mathrm{BD}$. Prove that $\triangle \mathrm{PAB}$ is similar to $\triangle \mathrm{PDC}$.

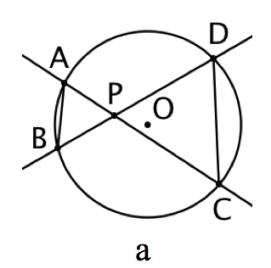

a

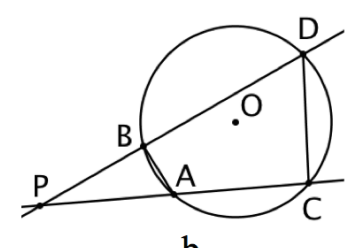

b

Figure 3: The proof problem and the local counterexample shown by the teacher (the first lesson)

The second lesson involved the problem in Figure 4, where the students proved the statement by showing $\angle \mathrm{PAC}=\angle \mathrm{PBD}$ and $\angle \mathrm{PCA}=\angle \mathrm{PDB}$ from the inscribed angle theorem. Contrary to the first lesson, the teacher then prompted the students to find local counterexamples of their proof by drawing various diagrams by themselves. The local counterexample typically discovered by the students was that shown in Figure 5a, where they found that the reason for $\angle \mathrm{PAC}=\angle \mathrm{PBD}$ was invalidated and needed to be changed to the inscribed quadrilateral theorem. The teacher introduced another local counterexample from a certain student's work (Figure 5b) where line AQ was a tangent line to circle O'. However, the class did not examine this case further because modification of the proof required the alternate angle theorem, which the students had not learnt.

As shown in the diagram on the right, two circles $\mathrm{O}$ and $\mathrm{O}$ ' intersect at points $\mathrm{P}$ and $\mathrm{Q}$, and two points $\mathrm{A}$ and $\mathrm{B}$ are located on circle $\mathrm{O}$. Point $\mathrm{C}$ is the intersection point of line AQ and circle $\mathrm{O}^{\prime}$, and point $\mathrm{D}$ is the intersection point of line $\mathrm{BQ}$ and circle $\mathrm{O}^{\prime}$. Prove that $\triangle \mathrm{PAC}$ is similar to $\triangle \mathrm{PBD}$.

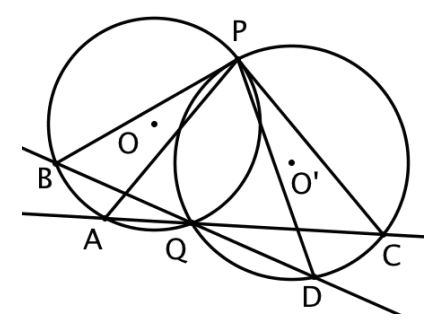

Figure 4: The proof problem (the second lesson) 


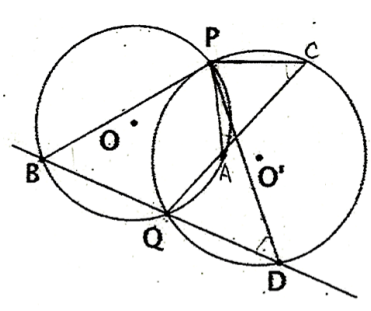

a

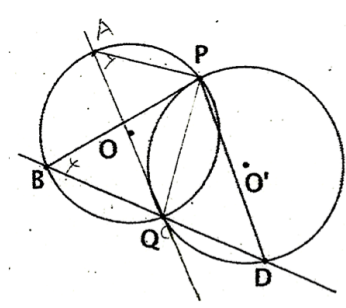

b

Figure 5: The local counterexamples from the students' worksheets (the second lesson)

\section{The third lesson}

The third lesson involved the proof problem in Figure 1, which differs slightly from the problem examined in the second lesson (Figure 4). We designed the assessment tasks, shown in Figure 6, to explore the research questions of this paper. These tasks have a hierarchical relationship; for example, students who cannot solve task 2 cannot give a correct answer to task 3 . These tasks correspond to the levels in the framework summarised in Table 1. Students are regarded at level 0 if they cannot solve any task or can solve task 1 only. Students are regarded at levels 1 and 2 if they can solve tasks 1-2 and tasks 1-3, respectively. Students are regarded at level 3 if they can solve tasks $1-3$, and further discover other kinds of local counterexamples.

Task 1: Take point $\mathrm{A}$ on various places on circle $\mathrm{O}$ and find a case that rejects your proof.

Task 2: Which part of your proof is rejected by this case?

Task 3: Modify your proof to show the similarity of $\triangle \mathrm{AQC}$ and $\triangle \mathrm{BPD}$ even in this case.

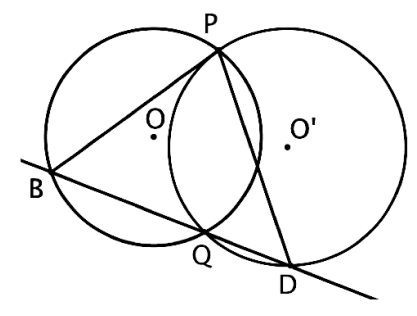

Figure 6: The assessment tasks

In the beginning of the third lesson, all the students proved the statement in Figure 1 by showing $\angle \mathrm{QAC}=\angle \mathrm{PBD}$ and $\angle \mathrm{QCA}=\angle \mathrm{PDB}$ from the inscribed angle theorem; students who found the proof difficult obtained help from other students. After that, the students worked individually at the tasks described in Figure 6 for twenty minutes. We obtained 27 students' responses, the classifications for which are shown in Table 2.

\begin{tabular}{lllll}
\hline & Level 0 & Level 1 & Level 2 & Level 3 \\
\hline The number of students & $11(41 \%)$ & $6(22 \%)$ & $4(15 \%)$ & $6(22 \%)$ \\
\hline
\end{tabular}

Table 2: The classification of the students' responses to the assessment tasks

Regarding the first research question in this paper, 16 students (59\% at levels $1-3)$ succeeded in proof validation, where they could draw diagrams that they recognised as local counterexamples invalidating their proof. The number of students at level 0 who failed in proof validation accounted for $41 \%$, which indicates that finding a local counterexample by drawing a diagram is difficult for many students even after explicit instruction.

The local counterexample discovered by the most students (12 students) was the case where point A was on arc PQ of circle O (Figure 7a). Five students drew the diagram where point $\mathrm{C}$ was on arc PQ 
of circle O' (Figure 2b). Some students found specific local counterexamples; e.g., three of the above 12 students put point A to be the intersection point of segment DP and circle $\mathrm{O}$ (Figure 7b), and three students drew the cases where line $\mathrm{AC}$ was a tangent line to circle $\mathrm{O}$ or $\mathrm{O}$ ' (Figure 7c). These answers seemed to be influenced by the second lesson, where specific diagrams such as Figure $5 \mathrm{~b}$ had been similarly presented. The number of local counterexamples discovered was larger than the number of students at levels 1-3 because several students discovered two or three local counterexamples.

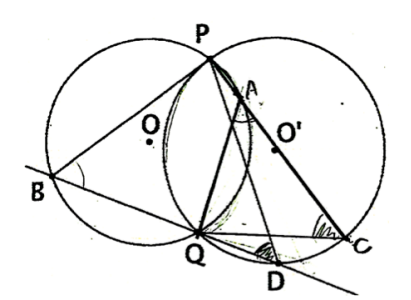

a

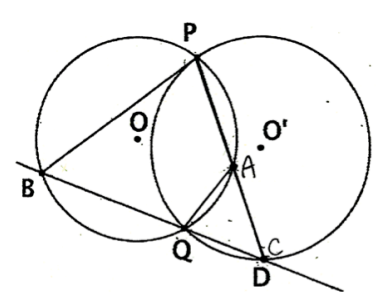

b

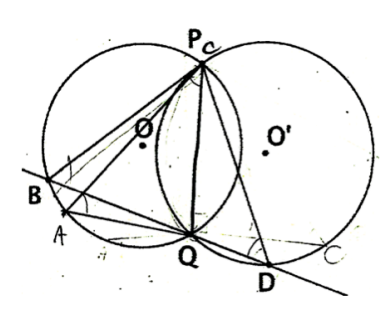

c

Figure 7: The local counterexamples from the students' worksheets (the assessment tasks)

Regarding the second research question in this paper, 10 students at levels 2 and 3 achieved proof modification, where they could adjust their proof to the local counterexamples they had discovered. This number accounts for $37 \%$ of all the students (10/27) and $63 \%$ of the students who succeeded in proof validation (10/16). Typical modification was an alteration in the reason for $\angle \mathrm{QAC}=\angle \mathrm{PBD}$ in Figure $7 \mathrm{a}$ from the inscribed angle theorem to the inscribed quadrilateral theorem. All the three students who drew Figure $7 \mathrm{~b}$ performed the same alteration and further changed the reason for $\angle \mathrm{QCA}=\angle \mathrm{PDB}$ from the inscribed angle theorem to the identity of these angles. The number of students at level 3 was larger than that at level 2, which indicates that discovering several local counterexamples might not be difficult for students who are successful in proof validation and modification.

Six students at level 2 achieved proof validation but not proof modification. Among them, three students drew the case in Figure $7 \mathrm{a}$, where they attempted to prove by changing the pair of angles from $\angle \mathrm{QAC}$ and $\angle \mathrm{PBD}$. For example, a student wrote incorrectly, "Since 4 points $\mathrm{P}$, B, Q, and A are inscribed in circle $\mathrm{O}, \angle \mathrm{BPA}=\angle \mathrm{AQC}$ ". One of the students at level 2 discovered the case in Figure $7 \mathrm{c}$, but failed in proof modification due to lack of knowledge of the alternate angle theorem. Other three students who drew the case in Figure 7c were regarded at level 4 because they found other local counterexamples wherein they succeeded in proof modification.

\section{DISCUSSION}

Contrary to the previous research focusing on graduate students, teachers, and mathematicians (e.g., Alcock \& Weber, 2005; Knuth, 2002; Weber, 2008), this paper investigated proof validation and modification by secondary school students. In the assessment tasks, $59 \%$ of the students succeeded in proof validation and $37 \%$ of the students succeeded in both proof validation and modification. Given the difficulty of the assessment tasks, the results are not disappointing. Japan has the national curriculum, which specifies that the inscribed quadrilateral theorem, which was necessary for proof modification in the tasks, be learnt in the tenth grade in upper secondary schools; the classroombased intervention in this study was implemented in the ninth grade in a lower secondary school. In 
addition, the results obtained by this intervention were better than those of our previous one-hour teaching experiment, implemented with a similar task (Komatsu \& Makino, 2014). In the future, a large-scale survey is necessary to scrutinise the possibility of generalising the results of this study derived from a single classroom.

The type of proof validation proposed in this paper, namely the discovery of local counterexamples by drawing diagrams, is an important activity in school mathematics. De Villiers (2010) examines the functions of experimentation that includes diagrammatic evaluation of conjectures and proofs, arguing that "we need to explore authentic, exciting and meaningful ways of incorporating experimentation and proof in mathematics education, in order to provide students with a deeper, more holistic insight into the nature of our subject" (p. 220). Lakatos (1976) rationally reconstructed the actual history of mathematics to demonstrate that "informal, quasi-empirical, mathematics does not grow through a monotonous increase of the number of indubitably established theorems but through the incessant improvement of guesses by speculation and criticism, by the logic of proofs and refutations" (p. 5). Therefore, proof validation and modification by example generation are essential in school mathematics to allow students to experience how mathematical knowledge progresses within its discipline.

As an implication for teaching, teachers may use proof problems with diagrams as tasks to introduce proof validation and modification into their classrooms in secondary schools. Although existing studies suggest that undergraduates' ability to comprehend and validate proofs could be improved by relevant instruction (e.g., Alcock \& Weber, 2005; Hodds, et al., 2014), they do not explore the kinds of tasks that were appropriate for the instruction. Our previous study demonstrated that proof problems with diagrams could provide students with an opportunity to experience refutation and generalisation of statements (Komatsu, et al, 2014). This paper complements the study by illustrating that these tasks can be used not only for the mathematical activity related to statements, but also for proof validation and modification.

There are two future research issues. First, the number of students at level 0 accounted for $41 \%$ in this study, indicating that the framework depicted in Table 1 has large meshes. If a case that constituted a local counterexample had been shown, some of the students could have pointed out and modified the refuted parts of their proof. It is necessary to develop a more fine-grained framework and assessment tasks for minutely capturing student proof validation and modification. Second, this study focuses on a peculiar type of proof validation in secondary school geometry. It is well known that some students make invalid proofs, such as a circular argument employing the conclusion as a hypothesis and overreliance on the appearance of a diagram to use conditions that are not given as hypotheses. It is necessary to present such invalid proofs and investigate whether students can invalidate and improve them.

\section{Acknowledgements}

This study is supported by the Japan Society for the Promotion of Science (Nos. 23330255 and 15H05402).

\section{References}

Alcock, L., \& Weber, K. (2005). Proof validation in real analysis: Inferring and checking warrants. The Journal of Mathematical Behavior, 24(2), 125-134. 
Common Core State Standards Initiative (2010). Common core state standards for mathematics. Retrieved August 27, 2015, from http://www.corestandards.org/wp-content/uploads/Math_Standards.pdf.

De Villiers, M. (2010). Experimentation and proof in mathematics. In G. Hanna, H. N. Jahnke, \& H. Pulte (Eds.), Explanation and proof in mathematics: Philosophical and educational perspectives (pp. 205-221). New York: Springer.

Hodds, M., Alcock, L., \& Inglis, M. (2014). Self-explanation training improves proof comprehension. Journal for Research in Mathematics Education, 45(1), 62-101.

Inglis, M., \& Alcock, L. (2012). Expert and novice approaches to reading mathematical proofs. Journal for Research in Mathematics Education, 43(4), 358-390.

Knuth, E. J. (2002). Secondary school mathematics teachers' conceptions of proof. Journal for Research in Mathematics Education, 33(5), 379-405.

Ko, Y. Y., \& Knuth, E. J. (2013). Validating proofs and counterexamples across content domains: Practices of importance for mathematics majors. The Journal of Mathematical Behavior, 32(1), 20-35.

Komatsu, K., \& Makino, K. (2014). Realizing explorative proving lessons in the unit "Using the relationship between inscribed angles and central angles" of grade 9. Journal of Japan Society of Mathematical Education, 96(9), 26-29. (in Japanese)

Komatsu, K., Tsujiyama, Y., Sakamaki, A., \& Koike, N. (2014). Proof problems with diagrams: An opportunity for experiencing proofs and refutations. For the Learning of Mathematics, 34(1), 36-42.

Lakatos, I. (1976). Proofs and refutations: The logic of mathematical discovery. Cambridge: Cambridge University Press.

Miyazaki, M., \& Fujita, T. (2015). Proving as an explorative activity in mathematics education: New trends in Japanese research into proof. In B. Sriraman, J. Cai, K. H. Lee, L. Fan, Y. Shimizu, C. S. Lim, \& K. Subramaniam (Eds.), The first sourcebook on Asian research in mathematics education: China, Korea, Singapore, Japan, Malaysia, and India - Singapore, Japan, Malaysia, and India Sections (pp. 13751407). Charlotte, NC: Information Age Publishing.

Powers, R. A., Craviotto, C., \& Grassl, R. M. (2010). Impact of proof validation on proof writing in abstract algebra. International Journal of Mathematical Education in Science and Technology, 41(4), 501-514.

Segal, J. (1999). Learning about mathematical proof: Conviction and validity. The Journal of Mathematical Behavior, 18(2), 191-210.

Selden, A., \& Selden, J. (2003). Validations of proofs considered as texts: Can undergraduates tell whether an argument proves a theorem? Journal for Research in Mathematics Education, 34(1), 4-36.

Selden, J., \& Selden, A. (1995). Unpacking the logic of mathematical statements. Educational Studies in Mathematics, 29(2), 123-151.

Simon, M. A. (1995). Reconstructing mathematics pedagogy from a constructivist perspective. Journal for Research in Mathematics Education, 26(2), 114-145.

Simon, M. A., \& Tzur, R. (2004). Explicating the role of mathematical tasks in conceptual learning: An elaboration of the hypothetical learning trajectory. Mathematical Thinking and Learning, 6(2), 91-104.

Stylianides, A. J., \& Stylianides, G. J. (2013). Seeking research-grounded solutions to problems of practice: Classroom-based interventions in mathematics education. ZDM - The International Journal on Mathematics Education, 45(3), 333-341.

Weber, K. (2008). How mathematicians determine if an argument is a valid proof. Journal for Research in Mathematics Education, 39(4), 431-459.

Weber, K. (2010). Mathematics majors' perceptions of conviction, validity, and proof. Mathematical Thinking and Learning, 12(4), 306-336. 